\title{
The Optimal Monthly Strategy Pricing of Free-Floating Bike Sharing Platform
}

\author{
Xi Cheng*, Yang Gao \\ School of Business Administration, South China University of Technology, Guangzhou, China \\ Email: *chengdaxi@foxmail.com
}

How to cite this paper: Cheng, X. and Gao, Y. (2018) The Optimal Monthly Strategy Pricing of Free-Floating Bike Sharing Platform. Modern Economy, 9, 318-338. https://doi.org/10.4236/me.2018.92021

Received: January 14, 2018

Accepted: February 24, 2018

Published: February 27, 2018

Copyright $\odot 2018$ by authors and Scientific Research Publishing Inc. This work is licensed under the Creative Commons Attribution International License (CC BY 4.0).

http://creativecommons.org/licenses/by/4.0/ cc) (i) Open Access

\begin{abstract}
This paper studies the revenue changes of the platform after the free-floating bike sharing platform adopts the monthly strategy, and obtains the best monthly subscription pricing to maximize the platform revenue. Through the comparison of user utility, we divide different types of users, depict the platform requirements, and thus find the platform revenue. We found that when the platform adopts the monthly strategy, if the monthly subscription price is too low, platform's revenue will be reduced. If the price is too high, the revenue will not change. Only in the case of a suitable monthly subscription price, platform revenue will increase. In addition, we find that the growth rate of platform revenue with the optimal pricing of monthly strategy is related to the purchase cost of bike. The lower the purchase cost, the higher the platform revenue growth rate.
\end{abstract}

\section{Keywords}

Sharing Economy, Free-Floating, Bike Sharing Platform, Pricing

\section{Introduction}

Business models that focus on providing access to assets rather than on transferring ownership of goods have become one of the most fundamental recent industry trends [1]. In the US automobile, for example, leasing has become increasingly important, reaching a market share of $27 \%$ in 2014 [2]. Similarly, bike sharing business models have become commonplace. The focus on enabling access rather than ownership is also one of the drivers of what has been called the sharing economy [3]. This movement from ownership-based to access-based business models has been very broad, involving sectors such as transportation (e.g., Uber, Mobike), accommodation and space (e.g., Airbnb, LiquidSpace), 
clothing (e.g., Tradesy, Kidizen), tools and household items (e.g., Streetbank, Peerby), and labor (e.g., Upwork, Fiverr).

Recent reports indicate that the five key sectors of sharing economic travel, product sharing, finance, staffing, music and video streaming are likely to increase global revenues from $\$ 1.5$ billion in 2015 to nearly $\$ 33.5$ billion in 2025 (Pricewaterhouse Coopers (PWC), 2015), and that $72 \%$ of Americans have engaged in transaction through the sharing economy or on-demand service companies [4]. Similarly, PWC establishes that $81 \%$ of consumers consider it less expensive to share goods than to own them individually, while $43 \%$ of consumers think "owing today feels like a burden" reflects the relevance of this spending trend. And this article is to study the first of the five major areas of sharing-traffic travel, and research on free-floating sharing vehicles.

Bike sharing systems are not new, since almost every major city from New York (e.g., Citi Bike) to London (e.g., Santander Cycles) to Paris (e.g., Vélib') has one. Unlike the station-based system in those cities where bike is kept at docks, the free-floating bike sharing systems allow them to be picked up and left anywhere in well-defined service regions, saving the last-mile walking from nearby bike stations to final destinations for riders. Thanks to the mobile applications for unlocking and tracking bikes, various bike sharing systems, especially free-floating ones such as Mobike and Ofo have brought more than 2 million new bikes to Chinese city streets [5]. Free-floating bike sharing systems are becoming more popular because of the flexibility in serving one-way trips. Latest reports show that Mobike's daily orders are more than 30 million, while Ofo's daily orders also exceeded 20 million; in terms of daily active users, the number of Mobike was 8.18 million, surpassing Ofo of 7.23 million [6]. With such a large user base and average daily orders, pricing strategies for sharing bicycles are even more important.

The motivation of this work is that we firmly believe that pricing policies are more important than ever, and that this is an essential part of the revenue management for bike sharing companies. The reason may be because price is one of the most effective variables managers can use to encourage or discourage demand.

In our last article, Free-floating vehicle sharing platform pricing strategy research [7], we mentioned that shared bicycles are priced according to the number of use, that is, 0.5 yuan or 1 yuan a time. Ofo charges 0.5 yuan a time while Mobike charges 1 yuan a time (Average each trip in half an hour, so made this approximate assumption). In July 2017, Ofo and Mobike launched monthly cards to encourage users to use shared bicycles monthly [8]. As we know, the pricing strategy is very important to the enterprise, especially to the enterprise that has tens of millions of daily orders for bike sharing platform. So what is the purpose of bike sharing platform to launch the monthly card? Just for profit to consumers? Can adopting the monthly strategy increase the platform revenue? This article will answer these questions by building mathematical models. 


\section{Literature Review}

Existing literature on sharing vehicles can be broadly divided into two categories according to whether the research problem is vehicle operation or revenue management. However, this paper studies the pricing strategy of free-floating vehicle sharing, belonging to the second category of income management. We will sort out the literature in accordance with these two categories.

One-way rentals pick up in one location and return at another, while round-way rentals pick up and return at the same location. In this article, free-floating vehicle sharing is a special case of one-way rentals. Let's review the literature on vehicle operation in one-way rentals.

One-way rentals in the operational literature can be divided into Data Analyzes, Rebalancing, Placement and Sizing of Stations three categories, respectively, as follows.

Data Analyses: The rich data on bike sharing systems has led to exploratory data analysis, trying to understand user behavior and congestion patterns, and trying to predict traffic between stations. Such work includes Nair et al. [9], Vogel and Mattfeld [10], Kaltenbrunner et al. [11], Lin and Yang [12], and Singhvi et al. [13].

Rebalancing: Fricker et al. [14] and Fricker and Gast [15] develop a stylized model of a bike sharing system to identify insights into bike deployment. An important observation is that in a balanced system with zero net flow at all stations, the total number of bicycles in the system should be slightly more than half the number of rackers, the difference being the number of bicycles used at any one time.

Placement and Sizing of Stations: García-Palomares et al. [16], Martinez et al. [17] and Romero et al. [18] aim to optimize the placement of stations in bike-sharing systems. Kabra et al. [19] uses statistical models to determine whether stations should be large and sparsely located, or small and densely located.

Due to the relatively new modes of free-floating vehicle sharing and the limited number of related documents, we collected as much as possible relevant literature on vehicle operation in free-floating vehicle sharing mode.

Similar to the traditional one-way rentals, the operational issues of free-floating vehicle sharing include Data Analyzes, Rebalancing (Relocation). However, because the free-floating vehicle sharing model allows customers to return their vehicle anywhere within the operation area, need not to rely on the depot stations. Therefore, the traditional Placement and Sizing of Stations problem will no longer be studied and replaced by the service region design issue.

Data Analyzes: Johannes and Stefan [20] get some conclusions that include user attributes and journey attributes are obtained by analyzing the booking data of trips in Berlin and Munich from 2013: free-floating vehicle sharing is mostly used by young well-educated people with an over-average income. The two main purposes of the trip are going home and spending free time activities. Svenja and 
Klaus [21] analyzed the demand impact factors of free-floating bike sharing system based on GPS-Data analysis of Munich's free-floating bike sharing system: The demand depends on different factors such as weather conditions, time of day and holidays/weekends.

Relocation: Similar to traditional one-way rentals, free-floating vehicle sharing is also faced with an imbalance of supply needs in the operating area, resulting in on one hand there zones, where a shortage of returned bikes occurs, no bikes are available but needed there, on the other hand there are zones, where too many bikes were returned but the demand for renting a bike there is low. A large number of scholars are devoted to studying the most effective relocation strategies and algorithms. Svenja and Klaus [21] give the application of an operator-based relocation strategy based on GPS-Data analysis of Munich's free-floating bike sharing system. By relocating at least some part of the fleet, it's ensured that demand for bikes is optimally satisfied in time and space. In the article of Simone and Klaus [22], different relocation algorithms for car sharing systems have been described, categorized and evaluated. In addition, the authors propose a new integrated two-step model for optimal vehicle positioning and relocation. Simone and Andrea [23] propose the Vehicle Distance Prediction approach to predict the distance of the nearest available vehicle at a given future instant, which shows vehicles could be moved in advance by the staff to balance the fleet distribute. Long and Zhenyu [24] study the fleet repositioning problem aiming to dynamically match the vehicle supply and travel demand at the lowest total cost of repositioning and lost sales. They develop a computationally efficient multi-stage robust model with enhanced linear decision rule.

Service Region Design: Long et al. [25] studied the planning issues faced by service providers in the design of service geographies to operate the service. This decision required maximizing customer traffic by covering travel needs and controlling fleet operating costs. They developed a mathematical programming model that combines the details of customer adoption and fleet management in an unbalanced travel model.

Next we organize the vehicle sharing revenue management related literature.

Van and Talluri [26] categorize revenue management as quantity-based if its primary tactical tool for managing demand is based on capacity-allocation decisions or price-based if it is based on prices.

Most previous research works on car rental focus on capacity controls (quantity-based revenue management), such as Conejero et al. [27], Gueeriero and Olivito [28], Haensel and Mederer [29], Steinhardt et al. [30].

In the following section, we will support the notion that dealing with pricing decisions is also important and sufficient for car rental companies and potentially adding some value to the discussion. First, the primary logical reasoning for volume-based revenue management will be based on two research processes. Then, we will introduce some work that tries to integrate or provide a common framework for dichotomous prices, such as Madden and Russell [31], they pro- 
posed an integer model based on the space-time network of rental locations. Each model had supply and demand relationships based on pricing levels for various vehicle types, optimized the choice of price levels, and relocated decisions.

This paper also studies the issue of revenue management for the quantity-price of shared vehicles, but differs from Madden and Russell [32] in that: 1) The research background is different: Madden and Russell [32] studied the traditional one-way rental, and this paper studies the free-floating sharing vehicle. Due to the traditional one-way rental site setting, the starting and ending points of consumers' expected journey will be affected by the location of the site. In the new mode of free-floating vehicle sharing, the expected journey of the consumer is consistent with the starting point of the actual journey, so that consumer utility is entirely determined by the distance traveled. 2) The research methods are different: Madden and Russell [32] developed a prototype rolling horizon linear programming model, and this paper builds a revenue model by drawing on the method of partitioning needs in the shared economy used by Jiang, Baojun and Lin [31]. 3) The purpose of the study is different: Madden and Russell [32] are committed to proposing a decision support tool to aid rental car companies in pricing and fleet capacity management decisions, and this article examines the dynamics of the business and analyzes the impact of the current price strategy of sharing vehicles on the revenue, consumer surplus and total social welfare of the platform companies. This article is dedicated to getting relevant management implications.

In the same area as my previous article, Free-floating vehicle sharing platform pricing strategy research [7], this paper focuses on the free-floating vehicle sharing pricing strategy research. But the difference is that the above studies are for car sharing platforms represented by Car2Go and bike sharing platform represented by Mobike, and compared the influence of fixed pricing strategy and distance-related pricing strategy on the two types of free-floating vehicle sharing platforms. This article focuses on bike sharing platform which adopt fixed pricing strategy, and analyzes the impact of the latest pricing strategy-the monthly strategy, on platform revenue.

\section{Model}

We consider a monopoly free-floating bike sharing platform (such as Mobike or Ofo) that provides users with a bike sharing service. Assuming that the user is heterogeneous in terms of the average number of trips per day and the average distance traveled per trip. For simplicity, we assume that users are evenly distributed in a square area of $[0,1] \times[0,1]$, let $\varepsilon \in[0,1]$ represent the average number of daily trips by the user, Where $\varepsilon=1$ represents the maximum average number of daily trips and $\varepsilon=0$ represents the minimum average number of daily trips. Let $\theta \in[0,1]$ represent the user's average travel distance each time, $\theta=1$ represents the maximum average travel distance per customer, $\theta=0$ 
represents the minimum average travel distance per user.

\subsection{The User Utility}

Each user has four choices: buy a bike, rent bikes and pay for usage, rent bikes and pay monthly, do not use (i.e., do not buy or rent) a bike. Let $U_{g}$ represents the utility a user chooses $g$, where $g=b$ represents the user buys a bike, $g=f$ represents the user rents bikes and pay for usage, $g=m$ represents the user rents bikes and pay monthly, $g=n$ represents the user do not use a bike. We assume $U_{n}=0$, that is, users have zero utility when not using a bike.

We assume that the utility users obtain each time they use the bike is proportional to the distance the user uses the bike. The reason is as follows. In general, users use bikes, there may be two main reasons: one is the need to use bikes to save time because of the urgency of time. The utility of the consumer is proportional to the time saved. If it is assumed that the user stays at a constant speed with the bike, the utility of the consumer is proportional to the distance used. The two is to get some kind of experience, such as a ride, a play, and so on, and the positive effect of the use of the bike experience is proportional to the use distance. In order to reduce the model parameters, we set the ratio coefficient is 1 , that is, the utility obtained by the consumer every time the vehicle is used equals the use distance.

\subsubsection{The Utility of Users Buying a Bike}

If a user buys a bike, the average daily cost of the bike purchased according to its life span will produce negative effects on the user. In general, the higher the proportion of the daily average cost of purchasing a bike to the daily per capita income of residents, the greater the negative effect of purchasing a bike. For the sake of analysis, we assume that the negative effect of purchasing a bike is equal to the ratio of the daily average cost of bikes to the daily per capita income of residents. Let the ratio be $c_{e}$, then $c_{e} \in[0,1]$. When $c_{e}$ is close to 1 , it indicates that the daily average cost of car purchase (sometimes referred to as bike costs without causing confusion) nearly accounts for the entire daily income per capita of residents. When $c_{e}$ is close to 0 , it means that the average daily cost of bike purchase accounts for a very small portion of daily income of residents. In this way, if consumers buy a bike, their average daily net utility equals the average number of bikes used per day multiplied by the average distance traveled per bike minus the daily average cost of purchased bikes as a percentage of residents' daily per capita income, that is:

$$
U_{b}=\varepsilon \times \theta-c_{e}
$$

\subsubsection{The Utility of Users Renting Bikes and Pay for Usage}

If the user does not buy a bike and chooses to rent a bike in free-floating bike sharing platform, due to the free parking of the users, the user may not be able to find the bike every time he wants to rent a bike. Suppose the probability that a user successfully found a bike each time is $a \in[0,1]$. In general, consumers' 
probability of finding a bike is positively related to the total number of bikes that the sharing platform launches in the consumer's area, therefore, $a$ can be regarded as the number of bikes in a certain area. Let $P$ represent the cost to be paid to the platform by the user, that is, the rental price of the shared bikes. Where $P_{f}$ represents the each payment a user renting bikes and pay for usage and $P_{m}$ represents the average daily rental price a user renting bikes and pay monthly. Then if the user chooses to rent bikes and pay for usage, the average daily net utility obtained is equal to the probability of successful rental to a bike multiplied by the average number of bikes used per day multiplied by the difference between each average ride distance and the pay-per-use rent to be paid to the platform, that is:

$$
U_{f}=a \times \varepsilon \times\left(\theta-P_{f}\right)
$$

\subsubsection{The Utility of Users Renting Bikes and Pay Monthly}

If the user chooses to rent bikes and pay monthly, the average daily net utility equals the probability of finding a successful bicycle multiplied by the average number of vehicles used per day multiplied by the average distance traveled per trip minus the average monthly cost to be paid to the platform, that is:

$$
U_{m}=a \times \varepsilon \times \theta-P_{m}
$$

Since we model from the platform perspective, we refer to category two and category three users as consumers.

\subsection{Platform's Revenue}

A platform with the probability that users successfully found a bike a, obtained revenue $a \times \varepsilon \times P_{f}$ from consumer who rents bikes and pay for usage and has $\varepsilon$ times a day on average, therefore, the revenue from all consumers who rent bikes and pay for usage is $\pi_{f}=\iint_{(\varepsilon, \theta) \in \Omega_{f}} a \times \varepsilon \times P_{f} \mathrm{~d} \varepsilon \mathrm{d} \theta$, where $\Omega_{f}$ is the set of consumers who rent bikes and pay for usage. The revenue bike sharing platform get from consumers who rent bikes and pay monthly is $P_{m}$, so the revenue from all consumers who rent bikes and pay monthly is $\pi_{m}=\iint_{(\varepsilon, \theta) \in \Omega_{m}} P_{m} \mathrm{~d} \varepsilon \mathrm{d} \theta$, where $\Omega_{m}$ is the set of consumers who rent bikes and pay monthly. So platform revenue is the sum of the two: $\pi=\pi_{f}+\pi_{m}$. As a preliminary study, this article will not discuss the issue of the number of bikes. But only focuses on the pricing of platforms under a certain capacity of bike. That is, the probability of finding a bike for each time $a$ is a constant, or can be understood as a pricing problem under which the platform maintains a fixed service level. Then the maximization of the platform's decision-making profit is equivalent to maximizing platform revenue:

$$
\pi=\pi_{f}+\pi_{m}=\iint_{(\varepsilon, \theta) \in \Omega_{f}} a \times \varepsilon \times P_{f} \mathrm{~d} \varepsilon \mathrm{d} \theta+\iint_{(\varepsilon, \theta) \in \Omega_{m}} P_{m} \mathrm{~d} \varepsilon \mathrm{d} \theta
$$

Because this paper is a simplified model of the business model of bike sharing 
reality, in reality, the sharing bike firstly introduces the pricing strategy of usage count, and then the monthly model is introduced based on the pricing strategy of usage count. Therefore, the bike sharing platform decision sequence is as follows: 1) When the user only has buy a bike, rent bikes and pay for usage, and do not use a bicycle, the three options, determine the optimal $P_{f}$ to maximize $\pi_{f} 2$ ) After joining the monthly strategy, the user has buy a bike, rent bikes and pay for usage, rent bikes and pay monthly, and do not use a bicycle, the four options. Then pricing by usage count $P_{f}$ does not change, determine the optimal $P_{m}$ for maximizing the revenue of the bike shared platform $\pi$.

\section{Analysis}

\subsection{Before a Monthly Strategy}

\subsubsection{Benchmark Model 1: No Bike Sharing (N)}

Firstly, we consider a no bike sharing scenario as a benchmark model. When there is no bike sharing, users only have two choices of buying and not buying, and there is no choice of renting. So when $U_{b}=\varepsilon \times \theta-c_{e} \geq 0$, users chose to buy a bike, and when $U_{b}=\varepsilon \times \theta-c_{e}<0$, users chose not to buy a bike. Accordingly, we can easily distinguish between users who buy and not buy a car. The result is shown in Figure 1, where the user in the gray shaded area is the user who buys the bike, and the user in the rest of the square is the user who does not buy or use the bike.

\subsubsection{Benchmark Model 2: Rent Bikes and Only Pay for Usage Pricing Strategy (F)}

When platforms provide renting bikes service and only pay for usage pricing strategy is available, consumers have three options: buying a bike, renting bikes and pay for usage, and not using a bike.

From the proposition 1 in Free-floating vehicle sharing platform pricing

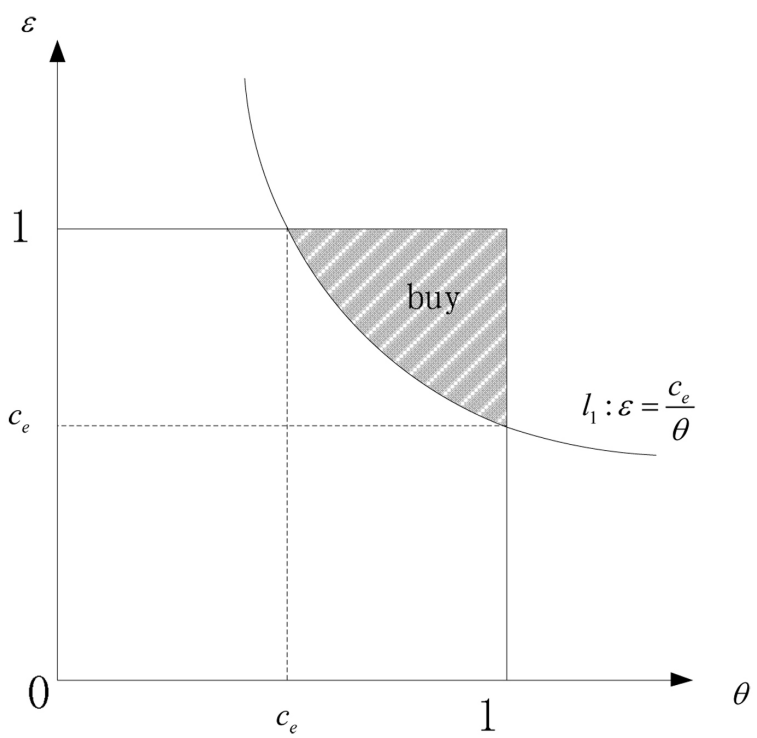

Figure 1. User groups in benchmark model 1. 
strategy research, we get the optimal pricing $P_{f}=p_{F}^{*}$ without monthly strategy: If $1-\frac{a}{2} \leq c_{e}<1$, then the optimal price of the vehicle sharing platform under the fixed pricing strategy is $p_{F}^{*}=\frac{1}{2}$; if $0 \leq c_{e}<1-\frac{a}{2}$, then the optimal price of the vehicle sharing platform under the fixed pricing strategy is $p_{F}^{*}=p_{F}^{s}<c_{e}$, Where $p_{F}^{s}$ is the only solution for

$L_{1}\left(p_{F}\right)=2\left(c_{e}-p_{F}\right)(1-a+a)^{2}-c_{e}^{2}(1-a)=0$ in interval $\left[0, c_{e}\right]$. Specific user groups as shown below.

With the optimal price $p_{F}^{*}$ under only pay for usage pricing strategy, the platform revenue is:

$$
\pi_{F}^{*}= \begin{cases}\pi_{F}^{s}=\frac{a p_{F}^{s}}{2(1-a)}\left(2 c_{e}-p_{F}^{s}-\frac{c_{e}^{2}}{1-a+a p_{F}^{s}}\right) & \text { when } 0 \leq c_{e}<1-\frac{a}{2} \\ \frac{a}{8}, & \text { when } 1-\frac{a}{2} \leq c_{e}<1\end{cases}
$$

\subsection{After a Monthly Strategy}

After determining the optimal pay-per-use, now we discuss the situation after the introduction of the monthly pricing strategy. When the platform offers consumers a monthly pricing strategy, there are four options for users to choose from: buy a bike, rent bikes and pay for usage, rent bikes and pay monthly, do not use a bike. According to the hypothesis of economic man, each user only belongs to one of the four groups of user.

\subsubsection{User Groups}

1) Group 1: users buying a bike

Only when the net utility of users buying a bike $U_{b}=\varepsilon \times \theta-c_{e} \geq 0$, and $U_{b}$ greater or equal to the net utility of renting bikes and pay for usage $U_{f}=a \times \varepsilon \times\left(\theta-P_{f}\right)$, and $U_{b}$ greater or equal to the net utility of renting bikes and pay monthly $U_{m}=a \times \varepsilon \times \theta-P_{m}$, users will choose to buy bikes. Then solve the inequality group:

$$
\left\{\begin{array}{l}
U_{b}=\varepsilon \times \theta-c_{e} \geq 0 \\
U_{b}=\varepsilon \times \theta-c_{e} \geq U_{f}=a \times \varepsilon \times\left(\theta-P_{f}\right) \\
U_{b}=\varepsilon \times \theta-c_{e} \geq U_{m}=a \times \varepsilon \times \theta-P_{m}
\end{array}\right.
$$

Then when $\max \left(\frac{c_{e}}{\theta}, \frac{c_{e}}{(1-a) \times \theta+a \times P_{f}}, \frac{c_{e}-P_{m}}{(1-a) \times \theta}\right) \leq \varepsilon \leq 1$, the user will choose to buy a bike.

2) Group 2: users renting bikes and pay for usage

Only when the net utility of users renting bikes and pay for usage $U_{f}=a \times \varepsilon \times\left(\theta-P_{f}\right) \geq 0$, and it greater or equal to the net utility of buying a bike $U_{b}=\varepsilon \times \theta-c_{e}$, and it greater or equal to the net utility of renting bikes pay monthly $U_{m}=a \times \varepsilon \times \theta-P_{m}$, users will choose to rent bikes and pay for usage. Then solve the inequality group: 


$$
\left\{\begin{array}{l}
U_{f}=a \times \varepsilon \times\left(\theta-P_{f}\right) \geq 0 \\
U_{f}=a \times \varepsilon \times\left(\theta-P_{f}\right) \geq U_{b}=\varepsilon \times \theta-c_{e} \\
U_{f}=a \times \varepsilon \times\left(\theta-P_{f}\right) \geq U_{m}=a \times \varepsilon \times \theta-P_{m}
\end{array}\right.
$$

Then when $0 \leq \varepsilon \leq \min \left(\frac{c_{e}}{(1-a) \times \theta+a \times P_{f}}, \frac{P_{m}}{a \times P_{f}}, 1\right)$ and $P_{f} \leq \theta \leq 1$, the consumer will choose to rent bikes and pay for usage.

3) Group 3: users renting bikes and pay monthly

Only when the net utility of users renting bikes and pay monthly $U_{m}=a \times \varepsilon \times \theta-P_{m} \geq 0$, and it greater or equal to the net utility of buying a bike $U_{b}=\varepsilon \times \theta-c_{e}$, and it greater or equal to the net utility of renting bikes pay for usage $U_{f}=a \times \varepsilon \times\left(\theta-P_{f}\right)$, users will choose to rent bikes and pay monthly. Then solve the inequality group:

$$
\left\{\begin{array}{l}
U_{m}=a \times \varepsilon \times \theta-P_{m} \geq 0 \\
U_{m}=a \times \varepsilon \times \theta-P_{m} \geq U_{b}=\varepsilon \times \theta-c_{e} \\
U_{m}=a \times \varepsilon \times \theta-P_{m} \geq U_{f}=a \times \varepsilon \times\left(\theta-P_{f}\right)
\end{array}\right.
$$

Then when $\max \left(\frac{P_{m}}{a \times \theta}, \frac{P_{m}}{a \times P_{f}}\right) \leq \varepsilon \leq \min \left(\frac{c_{e}-P_{m}}{(1-a) \times \theta}, 1\right)$, the consumer will choose to rent bikes and pay monthly.

As we know, group 2 and group 3 are consumers of bike sharing platforms.

4) Users not use a bike

Only when the net utility of users buying a bike $U_{b}=\varepsilon \times \theta-c_{e}<0$, and the net utility of renting bikes and pay for usage $U_{f}=a \times \varepsilon \times\left(\theta-P_{f}\right)<0$, and the net utility of renting bikes and pay monthly $U_{m}=a \times \varepsilon \times \theta-P_{m}<0$, users will choose not to buy or rent bikes. Then solve the inequality group:

$$
\left\{\begin{array}{l}
U_{b}=\varepsilon \times \theta-c_{e}<0 \\
U_{f}=a \times \varepsilon \times\left(\theta-P_{f}\right)<0 \\
U_{m}=a \times \varepsilon \times \theta-P_{m}<0
\end{array}\right.
$$

Then when $0 \leq \varepsilon \leq \min \left(\frac{c_{e}}{\theta}, \frac{P_{m}}{a \times \theta}, 1\right)$ and $\theta<P_{f}$, the user will choose not to buy or rent a bike.

\subsubsection{Platform's Revenue}

In order to get the platform's revenue function, we need to further identify four types of user groups. That is to say, it is necessary to determine relative position relationship of the demarcation line between users who buy bikes (group 1) and not use (group 4) $l_{1}: \varepsilon=\frac{c_{e}}{\theta}$, the demarcation line between group 1 and users who rent bikes and pay for usage (group 2) $l_{2}: \varepsilon=\frac{C_{e}}{(1-a) \times \theta+a \times P_{f}}$, and the demarcation line between group 2 and group $4 l_{3}: \theta=P_{f}$, the demarcation line 
between group 1 and users who rent bikes and pay monthly (group 3) $l_{4}: \varepsilon=\frac{C_{e}-P_{m}}{(1-a) \times \theta}$, the demarcation line between group 2 and group 3 $l_{5}: \varepsilon=\frac{P_{m}}{a \times P_{f}}$, the demarcation line between group 4 and group $3 l_{6}: \varepsilon=\frac{P_{m}}{a \times \theta}$, within the user group area $[0,1] \times[0,1]$. To do this, let $\theta$ be the horizontal axis and $\varepsilon$ the vertical axis as the plane $\theta-\varepsilon$, then all users fall within the square $[0,1] \times[0,1]$ in the first quadrant of the $\theta-\varepsilon$ plane.

As the expression of $l_{1}, l_{2}, l_{3}$ are not related to $P_{m}$, the user group with the optimal pricing $P_{f}$ is shown in Figure 2 and Figure 3. Therefore, based on Figure 2 and Figure 3, we continue our discussion of the relative position of $l_{4}, l_{5}, l_{6}$.

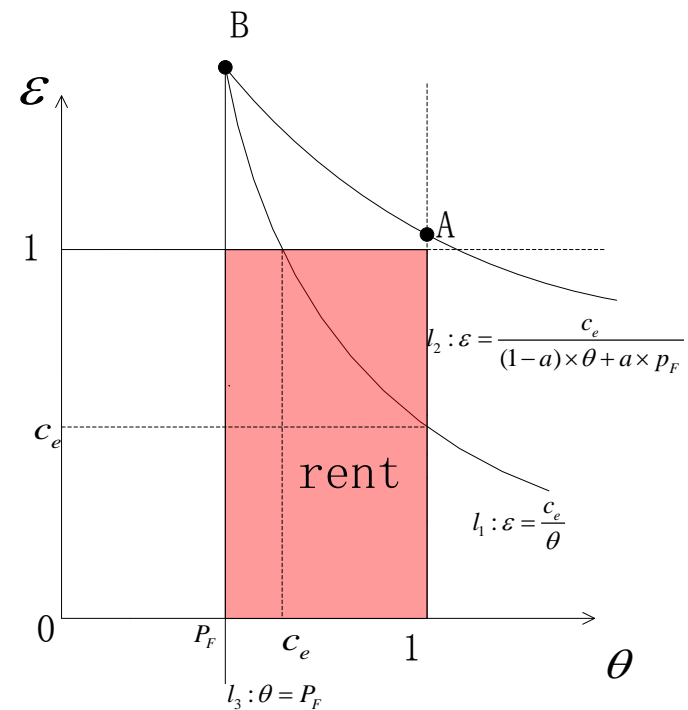

Figure 2. User groups in benchmark model 2 (when $\left.1-\frac{a}{2} \leq c_{e}<1\right)$.

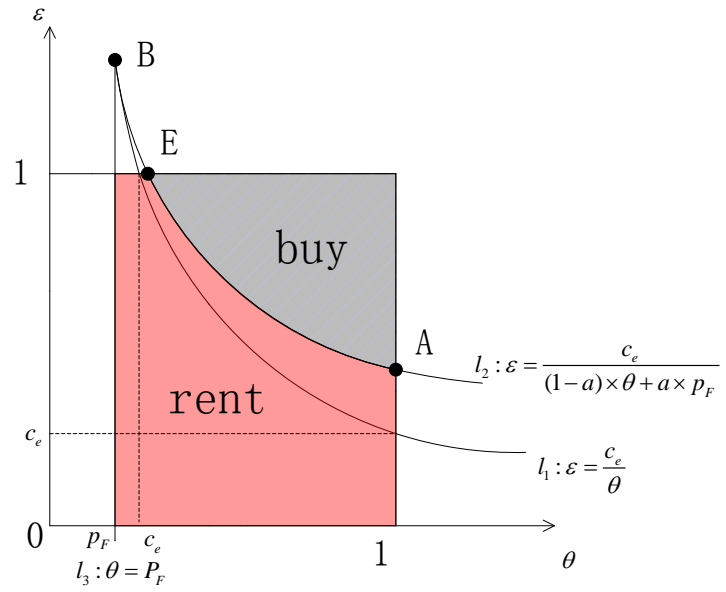

Figure 3. User groups in benchmark model 2 (when $\left.0 \leq c_{e}<1-\frac{a}{2}\right)$. 
From the six dividing line equation is easy to know, the three demarcation lines $l_{3}, l_{5}, l_{6}$ intersect at point $G\left(P_{f}, \frac{P_{m}}{a P_{f}}\right)$.

1) when $1-\frac{a}{2} \leq c_{e}<1$

$1-\frac{a}{2} \leq c_{e}<1$, then $P_{f}=\frac{1}{2}<\frac{c_{e}+a-1}{a}$, the utility of renting bikes paying for usage is always greater than the utility of buying a bike. Therefore, when adding the option of renting bikes paying monthly, the user will not choose to buy a bicycle either. So when $1-\frac{a}{2} \leq c_{e}<1$, users would choose among three options: renting a bike paying for usage, renting a bike by paying monthly and not using a bike. The introduction of the monthly pricing strategy, in fact, increased the platform's internal competition.

1) when $P_{m}>a P_{f}$, there is $U_{f}=a \times \varepsilon \times\left(\theta-P_{f}\right)>U_{m}=a \times \varepsilon \times \theta-P_{m}$. That is, the utility of renting bikes paying for usage is always greater than the utility of renting bikes paying monthly. Therefore, the paying for usage option is always better than the one paying monthly. So when $P_{m}$ is high, the newly added monthly rent-a-bike option will not be accepted by users. Consumption division and platform revenue are consistent with the benchmark model 2 . That is why we focus on the situation that $0 \leq P_{m} \leq a P_{f}$.

2) when $0 \leq P_{m} \leq a P_{f}$, We can easily judge the relative position of the three demarcations $l_{3}, l_{5}, l_{6}$ as shown in Figure 4 . In this way, based on the above analysis, we can easily know that the users who fall within the area enclosed by $l_{5}, l_{6}$ and boundary lines $\theta=1$ and $\varepsilon=1$ (i.e. the yellow shaded area in Figure 4) will choose to rent bikes paying monthly, and the users who fall within the area enclosed by $l_{3}, l_{5}$ and boundary lines $\theta=1, \varepsilon=\frac{P_{m}}{a P_{f}}$ (i.e. the red

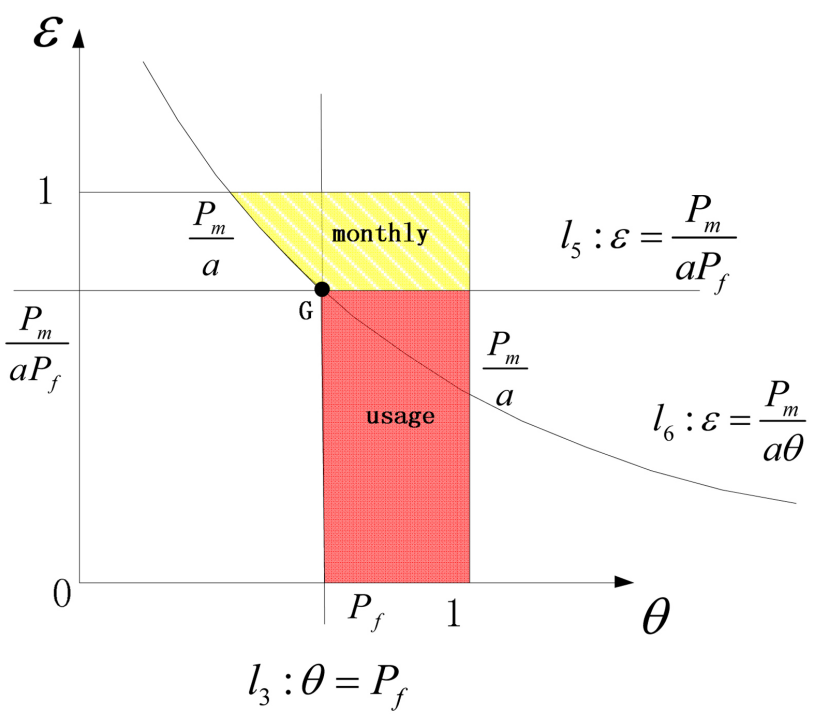

Figure 4. User groups in mode 1. 
shaded area in Figure 4) will choose to rent bikes paying per use, and the users who fall within the rest blank area in the square area will choose not to buy or rent a bike.

Then, the revenue of platform under $1-\frac{a}{2} \leq c_{e}<1$ and $0 \leq P_{m} \leq a P_{f}$ is:

$$
\begin{aligned}
\pi_{m s 1} & =\pi_{f m s 1}+\pi_{m m s 1}=\int_{P_{f}}^{1} \int_{0}^{P_{m} / a P_{f}} a \times P_{f} \times \varepsilon \mathrm{d} \varepsilon \mathrm{d} \theta+\int_{P_{m} / a P_{f}}^{1} \int_{p_{m} / a \varepsilon}^{1} P_{m} \mathrm{~d} \theta \mathrm{d} \varepsilon \\
& =\frac{P_{m}^{2}\left(1-P_{f}\right)}{2 a p_{f}}+P_{m}-\frac{P_{m}^{2}}{a p_{f}}+\frac{P_{m}^{2}}{a} \ln \frac{P_{m}}{a P_{f}}
\end{aligned}
$$

Take the derivative of $\pi_{m s 1}$ with respect to $P_{m}$, we found $\frac{\mathrm{d} \pi_{m s 1}}{\mathrm{~d} P_{m}}>0$. Therefore, the best platform income is obtained on the right boundary, $P_{m}^{*}=a P_{f}=\frac{a}{2}$, and then $\pi_{m s 1}^{*}=\frac{a}{8}=\pi_{F}^{*}$ which means when $1-\frac{a}{2} \leq c_{e}<1$, if $0 \leq P_{m} \leq a P_{f}$, then $\pi_{m s 1}^{*}<\pi_{F}^{*}$; if $P_{m} \geq a P_{f}$, then $\pi_{m s 1}^{*}=\pi_{F}^{*}$. So, we get Proposition 1 .

Proposition 1: when $1-\frac{a}{2} \leq c_{e}<1$, launching a monthly rental pricing strategy does not increase the platform's revenue, appropriate pricing can only make the income flat with before. What's worse, low pricing will lead to lower revenue. Therefore, when $1-\frac{a}{2} \leq c_{e}<1$, if only from the purpose of increasing revenue, it is not recommended to launch a monthly bike sharing pricing strategy.

That's because when $1-\frac{a}{2} \leq c_{e}<1$, there are no users buying a bike, and the utility of the user renting bikes paying for usage is already high. In order to allow users to shift from pay-per-use to monthly, platform must make the monthly rental price low enough, in order to make the user a higher monthly rent utility. However, the internal competition through price cuts will make the platform lower revenue. In order to avoid internal friction, when bike capacity and bike costs are both high, platform should maintain its status quo, not to launch a monthly strategy.

2) when $0 \leq c_{e}<1-\frac{a}{2}$

$0 \leq c_{e}<1-\frac{a}{2}$, then $P_{f}=p_{F}^{s}<c_{e}$. Same as in 1$)$, the paying for usage option is always better than the one paying monthly. Consumption division and platform revenue are consistent with the benchmark model 2. So, we get Proposition 2.

Proposition 2: The lower bound of the platform's maximum revenue is $\pi_{F}^{*}$. That is, introducing monthly strategy, as long as the pricing is reasonable, platform revenue will be higher than before, or at least equal with the previous revenue.

Then we focus on the situation when $0 \leq p_{m} \leq a p_{f}$.

1) Mode 1. When $0 \leq c_{e}<1-\frac{a}{2}, P_{m} \in\left[0, c_{e}+a-1\right]$, then 
$U_{m}=a \times \varepsilon \times \theta-P_{m} \geq U_{b}=\varepsilon \times \theta-c_{e}$. The utility of renting bikes and pay monthly is always greater than that of a user buying a bike. Therefore, no user will choose to buy a bike, and the user selects among the three options of renting bikes and pay for usage, renting on a monthly basis, and not using a bike, just like Figure 4 in 1). Optimal pricing of the platform with the largest revenue is on the right end, that is, $P_{m}^{*}=c_{e}+a-1$,

$$
\pi_{m s 1}=\frac{\left(c_{e}+a-1\right)^{2}\left(1-P_{f}\right)}{2 a P_{f}}+\left(c_{e}+a-1\right)-\frac{\left(c_{e}+a-1\right)^{2}}{a P_{f}}+\frac{\left(c_{e}+a-1\right)^{2}}{a} \ln \frac{c_{e}+a-1}{a P_{f}} .
$$

2) Mode 2. when $P_{m} \in\left[\max \left(0, c_{e}+a-1\right), \frac{a c_{e} P_{f}}{1-a+a P_{f}}\right]$, we know

$\frac{c_{e}-P_{m}}{1-a}>\frac{c_{e}}{1-a+a P_{f}}>\frac{c_{e}-P_{f}}{1-a}$ and $\frac{c_{e}-P_{m}}{1-a}>\frac{P_{m}}{a P_{f}}$. We can easily judge the relative position of the six demarcations $l_{1}, l_{2}, l_{3}, l_{4}, l_{5}, l_{6}$, and found that the division of the user groups division lines are $l_{3}, l_{4}, l_{5}, l_{6}$ as shown in Figure 5 . In this way, based on the above analysis, we can easily know that the users who fall within the area enclosed by the curve $l_{4}$ and boundary lines $\theta=1$ and $\varepsilon=1$ (i.e. the gray shaded area in Figure 5) will choose to buy a bike, and the users who fall within the area enclosed by the curve $l_{4}, l_{6}$ and $l_{5}$ and boundary lines $\theta=1$ and $\varepsilon=1$ (i.e. the yellow shaded area in Figure 5) will choose to rent bikes paying monthly, and the users who fall within the area enclosed by $l_{3}, l_{5}$ and boundary lines $\theta=1$ and $\varepsilon=\frac{P_{m}}{a P_{f}}$ (i.e. the red shaded area in Figure 5) will choose to rent bikes paying for usage, and the users who fall within the rest blank area in the square area will choose not to buy or rent a bike.

We can get the platform's revenue:

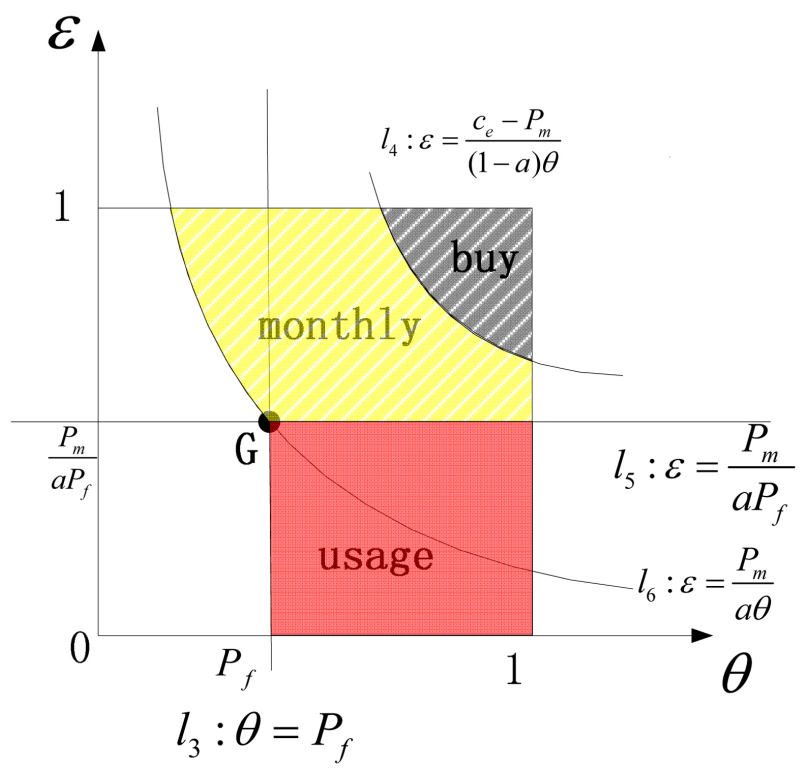

Figure 5. User groups in mode 2. 


$$
\begin{aligned}
\pi_{m s 2}= & \pi_{f m s 2}+\pi_{m m s 2} \\
= & \int_{p_{f}}^{1} \int_{0}^{p_{m} / a p_{f}} a \times p_{f} \times \varepsilon \mathrm{d} \varepsilon \mathrm{d} \theta+\int_{p_{m} / a p_{f}}^{1} \int_{p_{m} / a \varepsilon}^{\left(c_{e}-p_{m}\right) /(1-a)} p_{m} \mathrm{~d} \theta \mathrm{d} \varepsilon \\
& +\int_{\left(c_{e}-p_{m}\right) /(1-a)}^{1} \int_{p_{m} / a p_{f}}^{\left(c_{e}-p_{m}\right) /(1-a) / \theta} p_{m} \mathrm{~d} \varepsilon \mathrm{d} \theta \\
= & \frac{p_{m}^{2}\left(1-p_{f}\right)}{2 a p_{f}}+p_{m}\left[\frac{p_{m}}{a} \ln \frac{p_{m}}{a p_{f}}-\frac{p_{m}}{a p_{f}}+\frac{c_{e}-p_{m}}{1-a}\left(1-\ln \frac{c_{e}-p_{m}}{1-a}\right)\right]
\end{aligned}
$$

Take the derivative of $\pi_{m s 2}$ with respect to $P_{m}$, we found $\frac{\mathrm{d}^{2} \pi}{\mathrm{d} P_{m}^{2}}<0$, and $\pi_{m s 2}$ in the interval $P_{m} \in\left[\max \left(0, c_{e}+a-1\right), \frac{a c_{e} P_{f}}{1-a+a P_{f}}\right]$ continuous, Therefore, $\pi_{m s 2}$ is concave. The optimal price is that make the first derivative equal to 0 , that is, $P_{m}^{*}=P_{m}^{s}<a P_{f}$, which $P_{m}^{s}$ is the solution of $\frac{\mathrm{d} \pi}{\mathrm{d} P_{m}}=\frac{2 P_{m}}{a} \ln \frac{P_{m}}{a P_{f}}-\frac{P_{m}}{a P_{f}}+\frac{c_{e}-P_{m}}{1-a}+\frac{2 P_{m}-c_{e}}{1-a} \ln \frac{c_{e}-P_{m}}{1-a}=0$.

3) Mode 3. when $P_{m} \in\left[\frac{a c_{e} P_{f}}{1-a+a P_{f}}, a P_{f}\right]$, we know $\frac{c_{e}}{1-a+a P_{f}}>\frac{c_{e}-P_{m}}{1-a}>\frac{c_{e}-a P_{f}}{1-a}$ and $\frac{P_{m}}{a P_{f}}>\frac{c_{e}}{1-a+a P_{f}}$. We can easily judge the relative position of the $l_{2}, l_{3}, l_{4}, l_{5}, l_{6}$ are as shown in Figure 6 , and find the three demarcation lines $l_{2}, l_{4}, l_{5}$ intersect at point $H\left(\frac{a P_{f}\left(c_{e}-P_{m}\right)}{(1-a) P_{m}}, \frac{P_{m}}{a P_{f}}\right)$. In this way, based on the above analysis, we can easily know that the users who fall within the area enclosed by the curve $l_{2}, l_{4}$ and boundary lines $\theta=1$ and $\varepsilon=1$ (i.e. the gray shaded area in Figure 6) will choose to buy a bike, and the users who fall within the area enclosed by the curve $l_{4}, l_{6}$ and $l_{5}$ and boundary lines $\varepsilon=1$ (i.e. the yellow shaded area in Figure 6) will choose to rent

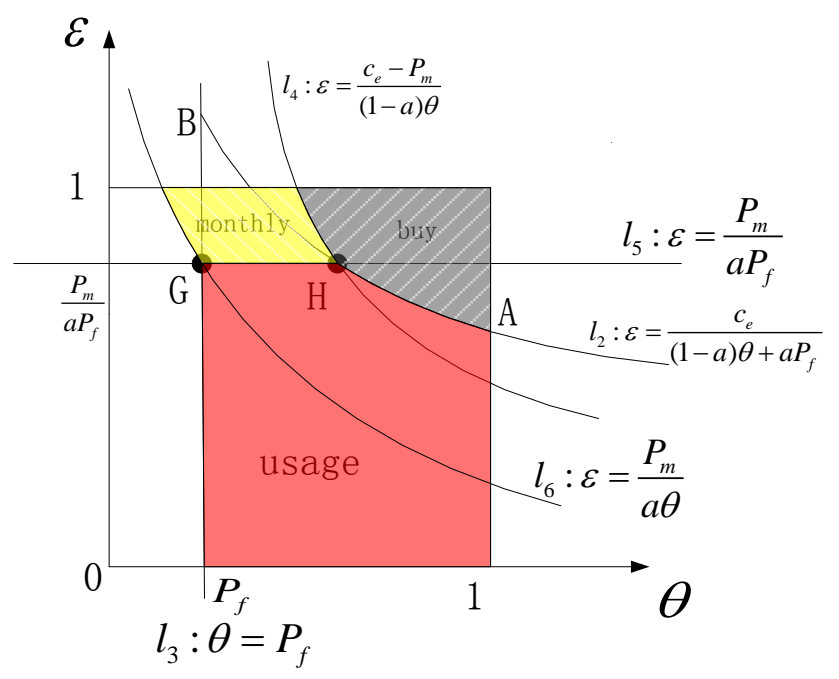

Figure 6. User groups in mode 3. 
bikes paying monthly, and the users who fall within the area enclosed by $l_{2}, l_{3}, l_{5}$ and boundary lines $\theta=1$ and $\varepsilon=\frac{P_{m}}{a P_{f}}$ (i.e. the red shaded area in Figure 5) will choose to rent bikes paying for usage, and the users who fall within the rest blank area in the square area will choose not to buy or rent a bike.

The platform's revenue consists of $\pi_{f}$ and $\pi_{m}$.

$$
\begin{aligned}
\pi_{f}=\int_{p_{f}}^{a p_{f}\left(c_{e}-p_{m}\right) /(1-a) / p_{m}} \int_{0}^{p_{m} / a p_{f}} a \times p_{f} \times \varepsilon \mathrm{d} \varepsilon \mathrm{d} \theta \\
+\int_{a p_{f}\left(c_{e}-p_{m}\right) /(1-a) / p_{m}}^{1} \int_{0}^{c_{e} /\left[(1-a) \theta+a p_{f}\right]} a \times p_{f} \times \varepsilon \mathrm{d} \varepsilon \mathrm{d} \theta \\
=a p_{f}\left[\frac{p_{m}^{2}}{2 a^{2} p_{f}^{2}}\left(\frac{a p_{f}\left(c_{e}-p_{m}\right)}{(1-a) p_{m}}-p_{f}\right)\right]+\frac{a c_{e}^{2} p_{f}}{2(1-a)}\left(\frac{p_{m}}{a p_{f} c_{e}}-\frac{1}{1-a+a p_{f}}\right) \\
=\frac{a p_{f}}{2(1-a)}\left[\frac{p_{m}\left(2 a c_{e}-p_{m}\right)}{a^{2} p_{f}}-\frac{1}{1-a+a p_{f}}\right] \\
\pi_{m}=\int_{p_{m} / a}^{p_{f}} \int_{p_{m} / a / \theta}^{1} p_{m} \mathrm{~d} \varepsilon \mathrm{d} \theta+\int_{p_{f}}^{\left(c_{e}-p_{m}\right) /(1-a)} \int_{p_{m} / a p_{f}}^{1} p_{m} \mathrm{~d} \varepsilon \mathrm{d} \theta \\
\quad+\int_{\left(c_{e}-p_{m}\right) /(1-a)}^{a p_{f}\left(c_{-}-p_{m}\right) /(1-a) / p_{m}} \int_{p_{m} / a p_{f}}^{\left(c_{e}-p_{m}\right) /(1-a) / \theta} p_{m} d \varepsilon d \theta \\
=p_{m}\left(\frac{c_{e}-p_{m}}{1-a}-\frac{p_{m}}{a}\right) \ln \frac{a p_{f}}{p_{m}} \\
\pi_{m s 3}=\pi_{f}+\pi_{m}=\frac{a p_{f}}{2(1-a)}\left[\frac{p_{m}\left(2 a c_{e}-p_{m}\right)}{a^{2} p_{f}}-\frac{c_{e}^{2}}{1-a+a p_{f}}\right] \\
\quad+p_{m}\left(\frac{c_{e}-p_{m}}{1-a}-\frac{p_{m}}{a}\right) \ln \frac{a p_{f}}{p_{m}}
\end{aligned}
$$

Take the derivative of $\pi_{m s 3}$ with respect to $P_{m}$, we get $\frac{\mathrm{d} \pi_{m s 3}}{\mathrm{~d} P_{m}}=\frac{2 P_{m}-a c_{e}}{a(1-a)} \ln \frac{P_{m}}{a P_{f}}$. Since $\ln \frac{P_{m}}{a P_{f}}<0$, the optimal price $P_{m}^{*}=\max \left(\frac{a c_{e}}{2}, \frac{a c_{e} P_{f}}{1-a+a P_{f}}\right)$.

In summary, when $1-\frac{a}{2} \leq c_{e}<1$, the largest revenue of platform $\pi=\max \left(\pi_{m s}, \pi_{m s 2}, \pi_{m s 3}\right)$. After comparing 1,2 and 3 we get the optimal monthly rental price that maximizes the platform revenue. Get the proposition 3.

Proposition 3. 1) when $1-a \leq c_{e}<1-\frac{a}{2}$, the optimal monthly rental price is $P_{m}^{*}=P_{m}^{s}<a P_{f}$, which $P_{m}^{s}$ is the only solution of $\frac{\mathrm{d} \pi}{\mathrm{d} P_{m}}=\frac{2 P_{m}}{a} \ln \frac{P_{m}}{a P_{f}}-\frac{P_{m}}{a P_{f}}+\frac{C_{e}-P_{m}}{1-a}+\frac{2 P_{m}-C_{e}}{1-a} \ln \frac{C_{e}-P_{m}}{1-a}=0$ in interval $\left[c_{e}+a-1, \frac{a c_{e} P_{f}}{1-a+a P_{f}}\right]$.2) when $0 \leq c_{e}<1-a$, the optimal price is 
$P_{m}^{*}=\max \left(\frac{a c_{e}}{2}, \frac{a c_{e} P_{f}}{1-a+a P_{f}}\right)$.

1) proof. When $1-a \leq c_{e}<1-\frac{a}{2}$, if $P_{m} \in\left[0, c_{e}+a-1\right]$, then $\frac{\mathrm{d} \pi_{m s 1}}{\mathrm{~d} P_{m}}>0$. if $P_{m} \in\left[\frac{a c_{e} P_{f}}{1-a+a P_{f}}, a P_{f}\right]$, then $\frac{\mathrm{d} \pi_{m s 3}}{\mathrm{~d} P_{m}}=\frac{2 P_{m}-a c_{e}}{a(1-a)} \ln \frac{P_{m}}{a P_{f}}<0$. Therefore, the

value is obtained in mode 2 .

The introduction of monthly pricing strategy by the platform may give rise to two outcomes for the user's demand. One is the positive impact that adding monthly pricing strategy will convert low-value users who did not previously use a bike to those who are in demand, or may have previously paid high-value users who originally intended to buy a bike to give up buying a bike and choose to rent bikes on a monthly basis. Both of these possibilities will increase the demand for bike sharing platform, and thereby increase the platform's revenue. Another is the negative impact, the introduction of the monthly strategy, in order to ensure the effectiveness of the strategy, the monthly price will be lower than the previous rental prices. The consumers' demand remains unchanged but consumers pay less, so that the platform's internal demand transformation will reduce the platform's revenue. In summary, the optimal pricing of monthly pricing is the trade-off between the two effects.

2) we know that when $0 \leq c_{e}<1-a$, the optimal monthly rental price is between $P_{m s 2}^{*}=P_{m s 2}^{s}$ and $P_{m s 3}^{*}$. The specific value depends on the value of these two platform revenue. We use numerical analysis found that all $\pi_{m s 2}^{*}$ are less than $\pi_{m s 3}^{*}$, with the following part of the data to indicate (Table 1).

Compare mode 2 and mode 3, we found that mode 2 attracts more monthly users while mode 3 has a higher unit monthly price. A comparison of $\pi_{m s 2}^{*}$ and $\pi_{m s 3}^{*}$ is actually a trade-off between demand and unit price. When $1-a \leq c_{e}<1-\frac{a}{2}$, demand dominates, while when $0 \leq c_{e}<1-a$ the opposite.

\section{Numerical Analysis}

After getting the maximum revenue, we compare the platform revenue after the monthly strategy with the previous one and we get the conclusion:

Proposition 4: When $0 \leq c_{e}<1-\frac{a}{2}$, the appropriate monthly pricing would result in increased platform revenue after the introduction of the monthly strategy.

When $1-\frac{a}{2} \leq c_{e}<1$, platform revenue will not increase, so the platform should maintain its status quo, no monthly strategy introduced, or platform can introduce an ineffective monthly strategy in order to make platform revenue does not reduce. When $0 \leq c_{e}<1-\frac{a}{2}$, if platform launch the monthly strategy, 
Table 1. Comparison between $\pi_{m s 2}^{*}$ and $\pi_{m s 3}^{*}$.

\begin{tabular}{|c|c|c|c|c|c|c|c|}
\hline$a$ & $c_{e}$ & $P_{f}$ & $\pi_{F}^{*}$ & $P_{m 2}^{*}$ & $\pi_{m s 2}^{*}$ & $P_{m 3}^{*}$ & $\pi_{m s 3}^{*}$ \\
\hline 0.3 & 0.1 & 0.093396 & 0.001859 & 0.002 & 0.000717 & 0.015 & 0.002002 \\
\hline 0.3 & 0.12 & 0.110624 & 0.002601 & 0.004 & 0.001474 & 0.018 & 0.002793 \\
\hline 0.3 & 0.14 & 0.127412 & 0.003441 & 0.006 & 0.002294 & 0.021 & 0.003683 \\
\hline 0.3 & 0.16 & 0.143775 & 0.004368 & 0.008 & 0.003182 & 0.024 & 0.004661 \\
\hline 0.3 & 0.18 & 0.159728 & 0.005372 & 0.01 & 0.004133 & 0.027 & 0.005716 \\
\hline 0.3 & 0.2 & 0.175282 & 0.006444 & 0.012 & 0.005141 & 0.03 & 0.006837 \\
\hline 0.3 & 0.22 & 0.190449 & 0.007575 & 0.016 & 0.006659 & 0.033 & 0.008016 \\
\hline 0.3 & 0.24 & 0.205241 & 0.008758 & 0.018 & 0.007765 & 0.036 & 0.009243 \\
\hline 0.3 & 0.26 & 0.219666 & 0.009982 & 0.022 & 0.009301 & 0.039 & 0.010509 \\
\hline 0.3 & 0.28 & 0.233733 & 0.011242 & 0.024 & 0.01048 & 0.042 & 0.011806 \\
\hline 0.3 & 0.3 & 0.247451 & 0.01253 & 0.028 & 0.012029 & 0.045 & 0.013127 \\
\hline 0.3 & 0.32 & 0.260826 & 0.013838 & 0.032 & 0.013556 & 0.048 & 0.014465 \\
\hline 0.3 & 0.34 & 0.273864 & 0.015161 & 0.034 & 0.0148 & 0.051 & 0.015811 \\
\hline 0.3 & 0.36 & 0.286572 & 0.01649 & 0.038 & 0.016319 & 0.054 & 0.01716 \\
\hline 0.3 & 0.38 & 0.298955 & 0.017821 & 0.042 & 0.017809 & 0.057 & 0.018506 \\
\hline 0.6 & 0.1 & 0.090305 & 0.005938 & 0.01 & 0.004318 & 0.03 & 0.006351 \\
\hline 0.6 & 0.12 & 0.106622 & 0.008184 & 0.016 & 0.006958 & 0.036 & 0.008723 \\
\hline 0.6 & 0.14 & 0.122517 & 0.010667 & 0.02 & 0.009302 & 0.042 & 0.011336 \\
\hline 0.6 & 0.16 & 0.138037 & 0.013349 & 0.026 & 0.012286 & 0.048 & 0.014148 \\
\hline 0.6 & 0.18 & 0.153223 & 0.016194 & 0.032 & 0.015388 & 0.054 & 0.017121 \\
\hline 0.6 & 0.2 & 0.168111 & 0.019168 & 0.04 & 0.01893 & 0.06 & 0.020223 \\
\hline 0.6 & 0.22 & 0.182731 & 0.022243 & 0.046 & 0.02219 & 0.066 & 0.023421 \\
\hline
\end{tabular}

appropriate monthly pricing will increase revenue. Because the monthly strategy mainly to attract high-value users who plan to buy a bike and the low value users who do not use the bike, increasing consumers' demand.

So, how much revenue can the platform increase with introduction a monthly strategy?

The calculation and statistics show that the average increase is $3.86 \%$ (Table 2).

Proposition 5. When $c_{e}$ is smaller, the rate of growth is larger; when $c_{e}$ is larger, the rate of growth is small.

Because the increase income of monthly strategy mainly from the transformation of the original purchase of bikes and people without the use of bike population, when $c_{e}$ is large, the purchase of the bike population itself is small, the market is occupied only by the shared bike platform rented by usage, and the increase of the consumption population is less. When the $c_{e}$ is less, the number of people buying a bike is more, if a monthly strategy is introduced, the number of people that can be transformed is bigger and more income is added. 
Table 2. Comparison of platform revenue before and after monthly policy implementation.

\begin{tabular}{ccccccc}
\hline$a$ & $c_{e}$ & $P_{f}$ & $\pi_{F}^{*}$ & $P_{m}^{*}$ & $\pi^{*}$ & growth rate \\
\hline 0.3 & 0.1 & 0.093396 & 0.001859 & 0.015 & 0.002002 & $7.69 \%$ \\
0.3 & 0.2 & 0.175282 & 0.006444 & 0.03 & 0.006837 & $6.10 \%$ \\
0.3 & 0.3 & 0.247451 & 0.01253 & 0.045 & 0.013127 & $4.76 \%$ \\
0.3 & 0.71 & 0.458482 & 0.035333 & 0.11 & 0.035654 & $0.91 \%$ \\
0.3 & 0.8 & 0.487129 & 0.037213 & 0.13 & 0.037298 & $0.23 \%$ \\
0.6 & 0.1 & 0.090305 & 0.005938 & 0.03 & 0.006351 & $6.96 \%$ \\
0.6 & 0.2 & 0.168111 & 0.019168 & 0.06 & 0.020223 & $5.50 \%$ \\
0.6 & 0.5 & 0.371015 & 0.06329 & 0.166 & 0.065272 & $3.13 \%$ \\
0.6 & 0.6 & 0.4353 & 0.071896 & 0.22 & 0.07289 & $1.38 \%$ \\
0.8 & 0.1 & 0.086174 & 0.013209 & 0.04 & 0.014035 & $6.25 \%$ \\
0.8 & 0.2 & 0.163435 & 0.037795 & 0.078 & 0.040102 & $6.10 \%$ \\
0.8 & 0.3 & 0.241877 & 0.062601 & 0.138 & 0.066176 & $5.71 \%$ \\
0.8 & 0.4 & 0.324166 & 0.082664 & 0.212 & 0.085546 & $3.49 \%$ \\
0.8 & 0.4 & 0.324166 & 0.082664 & 0.214 & 0.085546 & $3.49 \%$ \\
0.8 & 0.5 & 0.410446 & 0.095543 & 0.302 & 0.096564 & $1.07 \%$ \\
\hline
\end{tabular}

\section{Conclusion}

1). Optimal pricing

When $1-\frac{a}{2} \leq c_{e}<1$, as long as $P_{m}^{*}$ is more than $a P_{f}$, it can ensure that income is not reduced. When $1-a \leq c_{e}<1-\frac{a}{2}$, the optimal pricing of vehicle shared platform rented by month is $P_{m}^{*}=P_{m}^{s}<a P_{f}$, and $P_{m}^{s}$ is the only solution of $\frac{\mathrm{d} \pi}{\mathrm{d} P_{m}}=\frac{2 P_{m}}{a} \ln \frac{P_{m}}{a P_{f}}-\frac{P_{m}}{a P_{f}}+\frac{C_{e}-P_{m}}{1-a}+\frac{2 P_{m}-C_{e}}{1-a} \ln \frac{C_{e}-P_{m}}{1-a}=0$ in the interval of $\left[c_{e}+a-1, \frac{a c_{e} P_{f}}{1-a+a P_{f}}\right]$. When $0 \leq c_{e}<1-a$, the optimal pricing is $P_{m}^{*}=\max \left(\frac{a c_{e}}{2}, \frac{a c_{e} P_{f}}{1-a+a P_{f}}\right)$. At this point, after the introduction of the monthly strategy, reasonable pricing can make the income increase, which is why free-floating vehicle platform later has launched a monthly strategy.

2) Revenue increases, related to $c_{e}$.

After the monthly strategy was introduced, the increase of platform revenue was related to $a$ and $c_{e}$. The average increase is $3.8 \%$, and $c_{e}$ is smaller, and greater increase is in the income ratio.

\section{References}

[1] Bardhi, F. and Eckhardt, G.M. (2012) Access-Based Consumption: The Case of Product Sharing. Journal of Consumer Research, 39, 881-898. 
https://doi.org/10.1086/666376

[2] Kessler, A.M. (2015) Auto Leasing Gains Popularity among American Consumers. The New York Times, 8 January 2015.

[3] Sundararajan, A. (2016) The Sharing Economy. The MIT Press, Cambridge.

[4] Cortese, A. (2016) A New Wrinkle in the Gig Economy: Workers Get Most of the Money. The New York Times, 20 July 2016.

[5] Financial Times (2017) Chinas Bike-Sharing Boom in Charts. http://www.ft.com/content/5efe95f6-0aeb-11e7-97d1-5e720a26771b

[6] Sohu Finance (2017) Ofo Claims 32 Million Orders a Day?

[7] Zhou, Y. and Cheng, X. (2017) Free-Floating Vehicle Sharing Platform Pricing Strategy Research. Working Paper.

[8] Sohu Technology (2017) 1 Yuan Monthly Card of Ofo May Cause Mobike’s Users to Reduce Again. http://www.sohu.com/a/168348337_229540

[9] Nair, R., et al. (2013) Large-Scale Vehicle Sharing Systems: Analysis of Velib. International Journal of Sustainable Transportation, 7, 85-106. https://doi.org/10.1080/15568318.2012.660115

[10] Vogel, P. and Mattfeld, D.C. (2011) Strategic and Operational Planning of Bike-Sharing Systems by Data Mining-A Case Study. Springer, Berlin, 127-141.

[11] Kaltenbrunner, A., et al., (2010) Urban Cycles and Mobility Patterns: Exploring and Predicting Trends in a Bicycle-Based Public Transport System. Pervasive and Mobile Computing, 6, 455-466. https://doi.org/10.1016/j.pmcj.2010.07.002

[12] Lin, J.-R. and Yang, T.-H. (2011) Strategic Design of Public Bicycle Sharing Systems with Service Level Constraints. Transportation Research Part E: Logistics and Transportation Review, 47, 284-294. https://doi.org/10.1016/j.tre.2010.09.004

[13] Singhvi, D., et al. (2015) Predicting Bike Usage for New York City's Bike Sharing System. 29th AAAI Conference on Artificial Intelligence, Austin, 25-30 January 2015, 110-114.

[14] Fricker, C., Gast, N. and Mohamed, H. (2012) Mean Field Analysis for Inhomogeneous Bike Sharing Systems. Discrete Mathematics and Theoretical Computer Science Proceedings, Montreal, Jul 2012, 365-376.

[15] Fricker, C. and Gast, N. (2014) Incentives and Redistribution in Homogeneous Bike-Sharing Systems with Stations of Finite Capacity. EURO Journal on Transportation \& Logistics, 5, 261-291.

[16] García-Palomares, J.C., et al. (2012) Optimizing the Location of Stations in Bike-Sharing Programs: A GIS Approach. Applied Geography, 35, 235-246. https://doi.org/10.1016/j.apgeog.2012.07.002

[17] Martinez, L.M., Caetano, L., Eir, T. and Cruz, F. (2012) An Optimization Algorithm to Establish the Location of Stations of a Mixed Fleet Biking System: An Application to the City of Lisbon. Procedia-Social and Behavioral Sciences, 54, 513-524. https://doi.org/10.1016/j.sbspro.2012.09.769

[18] Romero, J.P., Ibeas, A., Moura, J.L., Benavente, J. and Alonso, B. (2012) A Simulation Optimization Approach to Design Efficient Systems of Bike-Sharing. Procedia-Social and Behavioral Sciences, 54, 646-655. https://doi.org/10.1016/j.sbspro.2012.09.782

[19] Kabra, A., Girotra, K. and Belavina, E. (2015) Bike-Share Systems: Accessibility and Availability.

[20] Johannes, M., Stefan, S. and Flemming, G. (2015) Identifying Users and Use of 
(Electric) Free-Floating Carsharing in Berlin and Munich. IEEE 18 th International Conference on Intelligent Transportation Systems, Las Palmas, 15-18 September 2015, 2568-2573.

[21] Reiss, S. and Bogenberger, K. (2015) GPS-Data Analysis of Munich's Free-Floating Bike Sharing System and Application of an Operator-Based Relocation Strategy. IEEE 18th International Conference on Intelligent Transportation Systems, Las Palmas, 15-18 September 2015, 584-589.

[22] Weikl, S. and Bogenberger, K. (2013) Relocation Strategies and Algorithms for Free-Floating Car Sharing System. IEEE Intelligent Transportation System Magazine, 5, 100-111. https://doi.org/10.1109/MITS.2013.2267810

[23] Formentin, S., Andrea, G., et al. (2015) On the Prediction of Future Vehicle Locations in Free-Floating Car Sharing Systems. IEEE Intelligent Vehicle Symposium, Seoul, 28 June-1 July 2015, 1006-1011. https://doi.org/10.1109/IVS.2015.7225816

[24] He, L., Hu, Z. and Zhang, M. (2017) Robust Repositioning for Vehicle Sharing. Working Paper.

[25] He, L., Mak, H.-Y., Rong, Y. and Max Shen, Z.-J. (2016) Service Region Design for Urban Electric Vehicle Sharing System. Working Paper.

[26] Ryzin Van, G.J. and Talluri, K.T. (2005) An Introduction to Revenue Management. In: Tutorials in Operations Research, INFORMS, 142-194.

[27] Conejero, J.A., Jordán, C. and Sanabria-Codesal, E. (2014) An Iterative Algorithm for the Management of an Electric Car-Rental Service. Journal of Applied Mathematics, 2014, Article ID: 483734. https://doi.org/10.1155/2014/483734

[28] Guerriero, F. and Olivito, F. (2014) Revenue Models and Policies for the Car Rental Industry. Journal of Mathematical Modelling and Algorithms in Operations Research, 13, 247-282. https://doi.org/10.1007/s10852-013-9234-8

[29] Haensel, A., Mederer, M. and Schmidt, H. (2012) Revenue Management in the Car Rental Industry: A Stochastic Programming Approach. Journal of Revenue and Pricing Management, 11, 99-108. https://doi.org/10.1057/rpm.2010.52

[30] Steinhardt, C. and Gönsch, J. (2012) Integrated Revenue Management Approaches for Capacity Control with Planned Upgrades. European Journal of Operational Research, 223, 380-391. https://doi.org/10.1016/j.ejor.2012.05.047

[31] Jiang, B. and Lin, T. (2016) Collaborative Consumption: Strategic and Economic Implications of Product Sharing. Management Science. https://doi.org/10.1287/mnsc.2016.2647

[32] Madden, T. and Russell, R.A. (2012) Modelling the Rental Car Pricing and Relocation Problem. International Journal of Revenue Management, 6, 246-257. https://doi.org/10.1504/IJRM.2012.050400 